\title{
Extended RPA with ground-state correlations in a solvable model
}

\author{
S. Takahara, M. Tohyama \\ Kyorin University School of Medicine, Mitaka, Tokyo 181-8611, Japan \\ P. Schuck \\ Institut de Physique Nucléaire, IN2P3-CNRS, Université Paris-Sud, F-91406 Orsay Cedex, France
}

(Dated: December 21, 2018)

\begin{abstract}
The ground states and excited states of the Lipkin model hamiltonian are calculated using a new theoretical approach which has been derived from an extended time-dependent Hartree-Fock theory known as the time-dependent density-matrix theory (TDDM). TDDM enables us to calculate correlated ground states, and its small amplitude limit (STDDM), which is a version of extended RPA theories based on a correlated ground state, can be used to calculate excited states. It is found that this TDDM plus STDDM approach gives much better results for both the ground states and the excited states than the Hartree-Fock ground state plus RPA approach.
\end{abstract}

The random phase approximation (RPA) based on the Hartree-Fock (HF) ground state and the quasi-particle RPA (QRPA) based on the Hartree-Fock-Bogoliubov (HFB) ground state when pairing correlations are important are standard microscopic and self-consistent theories which have extensively been used to study nuclear collective excitations [1]. RPA and QRPA are regarded as mean-field theories because they can be formulated as the small amplitude limits of the time-dependent HF theory (TDHF) and the time-dependent HFB theory, respectively. On the other hand the time-dependent density-matrix theory (TDDM) 2] which we have developed in the past decade incorporates the effects of two-body correlations into TDHF, and its small amplitude limit (STDDM) 3] becomes a natural extension of RPA which includes two-body amplitudes and also the effects of ground-state correlations: The correlated ground state is obtained as a stationary solution of the TDDM equations. We have recently applied this TDDM plus STDDM approach to study the ground states and low-lying quadrupole states in unstable oxygen isotopes [4, 5]. Although those results are quite encouraging, the validity of the TDDM plus STDDM approach should be tested in further applications. In this letter, we apply TDDM and STDDM to the Lipkin model and calculate the ground states and excited states. We will show that TDDM and STDDM give much better results than HF and RPA.

The correlated ground state $\left|\Phi_{0}\right\rangle$ in TDDM is constructed such that the two equations,

$$
\begin{gathered}
F_{1}(\alpha \beta)=\left\langle\Phi_{0}\left|\left[a_{\alpha}^{+} a_{\beta}, H\right]\right| \Phi_{0}\right\rangle=0, \\
F_{2}\left(\alpha \beta \alpha^{\prime} \beta^{\prime}\right)=\left\langle\Phi_{0}\left|\left[a_{\alpha}^{+} a_{\beta}^{+} a_{\beta^{\prime}} a_{\alpha^{\prime}}, H\right]\right| \Phi_{0}\right\rangle=0,
\end{gathered}
$$

are satisfied, where [ ] is the commutation relation and $H$ is the total hamiltonian consisting of the kinetic energy term and a two-body interaction. Eqs.(11) and (2) describe the conditions that the occupation matrix $n_{\alpha \beta}^{0}=\left\langle\Phi_{0}\left|a_{\beta}^{+} a_{\alpha}\right| \Phi_{0}\right\rangle$ and the two-body correlation matrix $C_{\alpha \beta \alpha^{\prime} \beta^{\prime}}^{0}=\left\langle\Phi_{0}\left|a_{\alpha^{\prime}}^{+} a_{\beta^{\prime}}^{+} a_{\beta} a_{\alpha}\right| \Phi_{0}\right\rangle-\mathcal{A}\left(n_{\alpha \alpha^{\prime}}^{0} n_{\beta \beta^{\prime}}^{0}\right)$, where $\mathcal{A}$ is the antisymmetrization operator, are time-independent. We choose the single-particle wavefunction $\psi_{\alpha}$ as an eigenstate of the mean-field hamiltonian $h_{0}\left(\rho_{0}\right)$ which is a functional of the one-body density matrix $\rho_{0}$. The expressions for Eqs.(11) and (2) are given in Ref. [5]. We have shown in Ref. [5] that Eqs.(11) and (2) can be solved using the iterative gradient method: Starting from the HF ground state where $n_{\beta \alpha}^{0}=\delta_{\beta \alpha}(0)$ for an occupied (unoccupied) state and $C_{\alpha \beta \alpha^{\prime} \beta^{\prime}}^{0}=0$, we iterate

$$
\left(\begin{array}{c}
n^{0}(N+1) \\
C^{0}(N+1)
\end{array}\right)=\left(\begin{array}{c}
n^{0}(N) \\
C^{0}(N)
\end{array}\right)-\alpha\left(\begin{array}{ll}
a & c \\
b & d
\end{array}\right)^{-1}\left(\begin{array}{l}
F_{1}(N) \\
F_{2}(N)
\end{array}\right)
$$

until convergence is achieved. The matrix elements $a, b, c$, and $d$, which are functional derivatives of $F_{1}$ and $F_{2}$ and depend on the iteration step $N$, are equivalent to those appearing in the hamiltonian matrix of STDDM. They are also given in Ref. [5]. We have to introduce a small parameter $\alpha$ to control the convergence process.

Excited states are obtained solving the STDDM equations for the one-body transition amplitude $x_{\alpha \beta}(\mu)=$ $\left\langle\Phi_{0}\left|a_{\beta}^{+} a_{\alpha}\right| \Phi_{\mu}\right\rangle$ and the two-body transition amplitude $X_{\alpha \beta \alpha^{\prime} \beta^{\prime}}(\mu)=\left\langle\Phi_{0}\left|a_{\alpha^{\prime}}^{+} a_{\beta^{\prime}}^{+} a_{\beta} a_{\alpha}\right| \Phi_{\mu}\right\rangle$, where $\left|\Phi_{\mu}\right\rangle$ is the wavefunction for an excited state with excitation energy $\omega_{\mu}$. The equations in STDDM can be written in matrix form

$$
\left(\begin{array}{ll}
a & c \\
b & d
\end{array}\right)\left(\begin{array}{l}
x \\
X
\end{array}\right)=\omega_{\mu}\left(\begin{array}{c}
x \\
X
\end{array}\right)
$$


When the ground-state $\left|\Phi_{0}\right\rangle$ is approximated by the HF one and only the particle (p) - hole (h) and $2 \mathrm{p}$ - 2h amplitudes (and their complex conjugates) are taken in Eq.(3), STDDM reduces to the second RPA (SRPA) [6]. The strength function $S(E)$ defined as

$$
S(E)=\sum_{\omega_{\mu}>0}\left|\left\langle\Phi_{\mu}|\hat{Q}| \Phi_{0}\right\rangle\right|^{2} \delta\left(E-\omega_{\mu}\right)
$$

for an excitation operator $\hat{Q}$ is given in terms of the solution of Eq.(3). The detailed expressions of $S(E)$ for one-body and two-body excitation operators are given in Ref. [5].

The Lipkin model [] describes an N-fermions system with two N-fold degenerate levels with energies $\epsilon / 2$ and $-\epsilon / 2$, respectively. The upper and lower levels are labeled by quantum number $p$ and $-p$, respectively, with $p=1,2, \ldots, N$. The model is described by the following hamiltonian

$$
H=\epsilon J_{z}+\frac{V}{2}\left(J_{+}^{2}+J_{-}^{2}\right)
$$

where the operators are given as

$$
\begin{array}{r}
J_{z}=\frac{1}{2} \sum_{p=1}^{N}\left(a_{p}^{+} a_{p}-a_{-p}{ }^{+} a_{-p}\right) \\
J_{+}=J_{-}^{+}=\sum_{p=1}^{N} a_{p}^{+} a_{-p} .
\end{array}
$$

Using an $N=4$ system as an example, we solve Eqs.(11) and (2) for the ground states and Eq.(3) for the excited states. The hamiltonian matrix in STDDM (Eq.(3)) is not hermitian in general [5, 8] and, as a result, there is a possibility that some eigenvalues become complex. However, the hamiltonian in the Lipkin model is so simple that all eigenvalues are real and that the strength functions are positive definite.

First we present the results for the ground states. We fix $\epsilon$ at 2 and change $V$. To obtain converged results in the iterative gradient method, we need to start with a small value of interaction strength $V$ and gradually increase it: We start with $V / 50$ and increase it by $V / 50$ for each 200 iterations. We found that the correlated ground states obtained coincide with those calculated using a time-dependent approach [9] which provides us with another method for obtaining a stationary solution of the TDDM equations. The total energies obtained in TDDM (dashed line) for various interaction strength $|V|$ are shown in Fig. 1 in comparison with the exact (solid line) and HF (dotted line) ones. The Lipkin model doesn't have stable HF ground states for $|V| / \epsilon>1 /(N-1)$. For $|V| / \epsilon>1 / 3$ in the case of $N=4$, the 'deformed' HF solution is shown [1, 7]. The results in TDDM and STDDM are always calculated using the original (not deformed) single-particle basis. The results in TDDM are very close to the exact ones in the wide range of the interaction strength: The deviation of the total energy from the exact one is only $1.1 \%$ at $|V| / \epsilon=0.3$.

Now we show the results for the first excited state (one-phonon state). We use $V=-0.6$ which is slightly smaller than the critical value $V=-2 / 3$. Fig. 2 shows the strength function for the excitation operator $\hat{Q}_{1}=J_{+}+J_{-}$ calculated in STDDM (dashed line). The strength functions for the exact solution (solid line) and the RPA solution (dotted line) are also drawn for comparison. The dot-dashed line depicts the result in a modified STDDM (mSTDDM) which will be discussed in connection with the two-phonon state. To make differences among various calculations easy to see, we smoothed the strength functions with an artificial width $\Gamma / \epsilon=0.025$. RPA overestimates the collectivity of the first excited state: The excitation energy is much lower than the exact solution and the transition strength becomes quite large. On the other hand both the excitation energy and the transition strength calculated in STDDM are very close to the exact values. There appears a state at $E / \epsilon=1.3$ in the STDDM result. This is due to the coupling to $3 \mathrm{p}-1 \mathrm{~h}$ and $1 \mathrm{p}-3 \mathrm{~h}$ configurations. The value of the energy-weighted sum $m_{1}$ of the transition strength is 14.2 in STDDM while the exact value is 14.6. The value of $m_{1}$ in RPA is 15.2. The results in STDDM demonstrate the importance of ground-state correlations and the coupling to the two-body amplitudes which are completely missing in RPA. The effects of ground-state correlations may be classified as the self-energy contributions, the modification of p-h interactions and the vertex corrections [10]. For example, the fact that the excitation energy in STDDM is much larger than that in RPA is due to the increase in the self-energy of $1 \mathrm{p}-1 \mathrm{~h}$ configurations. However, it is not easy in our approach to clearly distinguish the various contributions of the ground-state correlations. The excitation energy of the one phonon state is shown in Fig 3 as a function of the interaction strength. It is clearly seen that STDDM avoids the breakdown of RPA in the region of the phase transition $(|V| / \epsilon>1 / 3)$ by including the effect of ground-state correlations. 


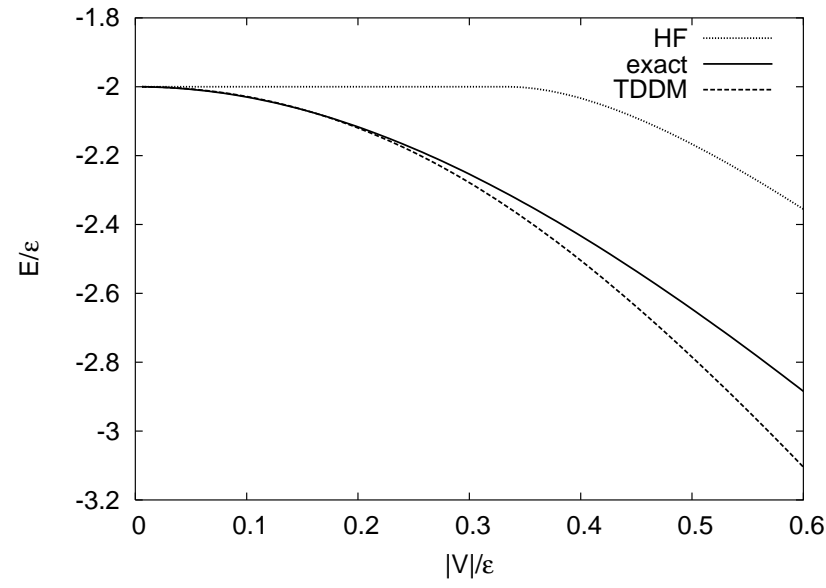

FIG. 1: Total energy $E / \epsilon$ as a function of $|V|$ for $N=4$. The solid, dashed, and dotted lines depict the exact solutions, the TDDM results, and the HF results, respectively.

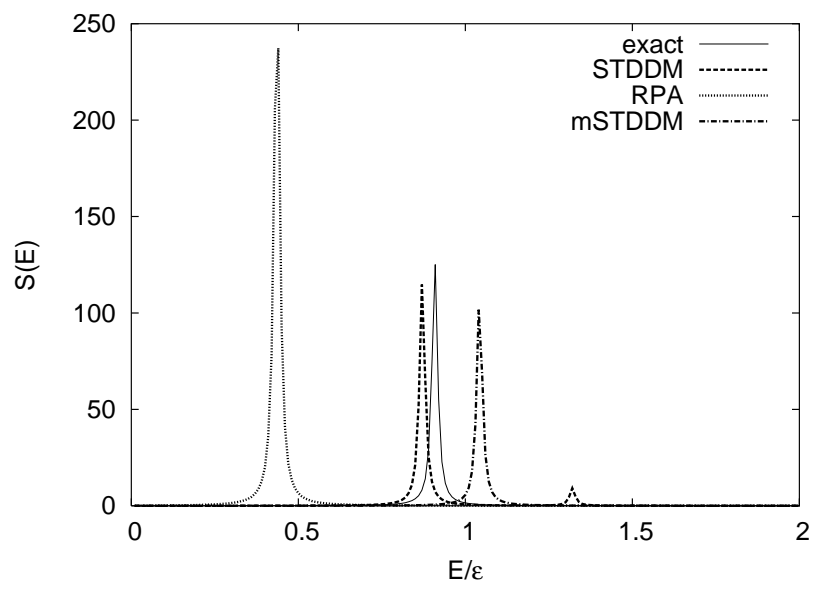

FIG. 2: Strength distributions of the one-phonon state calculated in STDDM (dashed line) and RPA (dotted line). The exact solution is shown with the solid line. The strength functions are smoothed with an artificial width $\Gamma / \epsilon=0.025$.

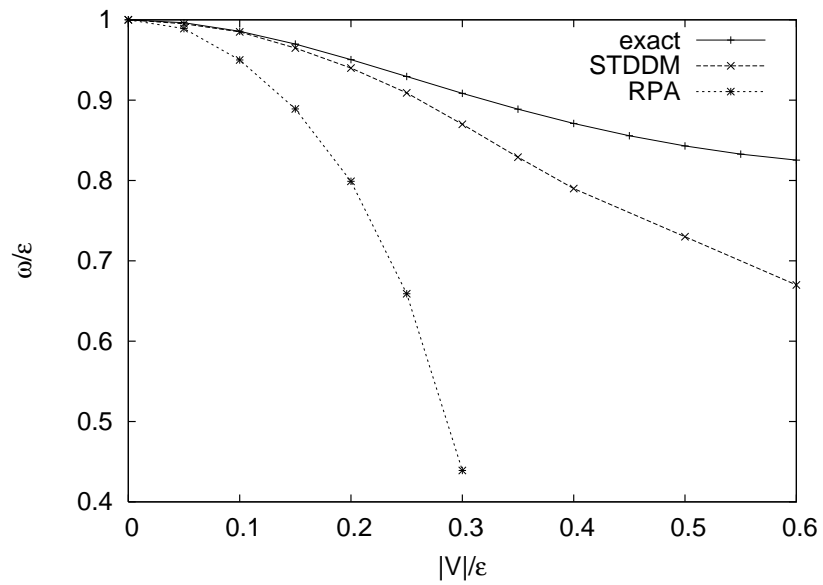

FIG. 3: Excitation energy of the first excited state as a function of the interaction strength. The dashed and dotted lines connect the results in TDDM and RPA, respectively, while the solid line indicates the exact solutions. 


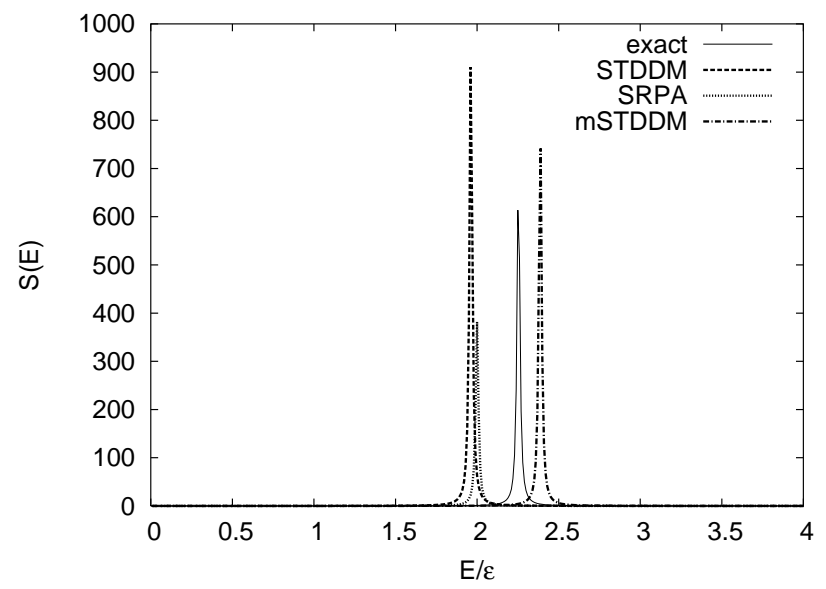

FIG. 4: Strength distribution of the two-phonon state of the Lipkin model calculated in STDDM (dashed line). The solid and dotted lines depict the exact solution and the result in SRPA, respectively. The result in mSTDDM is shown with the dot-dashed line. The strength functions are smoothed with an artificial width $\Gamma / \epsilon=0.025$.

The strength function in STDDM (dashed line) for the two-phonon state excited with $\hat{Q}_{2}=\left(J_{+}+J_{-}\right)^{2}$ is shown in Fig. 4 where the strength function of the exact solution (solid line) and that in SRPA (dotted line) are also drawn for comparison. The interaction strength used is $V=-0.6$. In SRPA where only $2 \mathrm{p}-2 \mathrm{~h}$ and $2 \mathrm{~h}-2 \mathrm{p}$ amplitudes are considered, neither coupling to the one-body amplitudes nor interaction among $2 \mathrm{p}-2 \mathrm{~h}$ configurations exist in the Lipkin model. Therefore, the result in SRPA is equivalent to the unperturbed one. The state in STDDM has much larger transition strength than SRPA. This is due to the fact that $X_{p p^{\prime} p^{\prime \prime} p^{\prime \prime \prime}}, X_{p h p^{\prime} h^{\prime}}$ and $X_{h h^{\prime} h^{\prime \prime} h^{\prime \prime \prime}}$ amplitudes in addition to $X_{p p^{\prime} h h^{\prime}}$ and $X_{h h^{\prime} p p^{\prime}}$ are taken into account in STDDM. However, the transition strength is overestimated and the excitation energy is lower than the exact solution. This is similar to the relation between the RPA result and the exact one for the one-phonon state. This means that the effects of ground-state correlations are not fully taken into account in STDDM for the two-phonon state. In fact, the self-energy terms for $2 \mathrm{p}-2 \mathrm{~h}$ configurations are not included in STDDM because they are kinds of three-body effects. In Ref. [5] we presented a prescription for taking into account the three-body effects in which missing terms are added to the hamiltonian matrix acting on the two-body amplitudes. (See Eq.(25) of Ref. [5] for a detailed explanation of the prescription.) We call this version of STDDM a modified STDDM (mSTDDM). The result in mSTDDM is shown in Fig. [4 with the dot-dashed line. It becomes closer to the exact solution: The transition strength is reduced and the excitation energy is increased. Due to the coupling to the two phonon state, the first excited state is slightly modified as well, as shown in Fig 2

In summary, we applied the TDDM plus STDDM approach to the Lipkin model. It was found that this approach gives much better results for the ground-state energies and the first excited states (the one-phonon state) than HF and RPA. However, the second excited state (two-phonon state) calculated in STDDM was not so good as the one-phonon state. This is due to the lack of some ground-state correlations coming from 3-body effects. It was shown that the modified STDDM which includes the three-body effects can improve the result for the two-phonon state.

[1] P. Ring and P. Schuck, The Nuclear Many-Body Problem, (Springer-Verlag, Berlin, 1980).

[2] M. Gong and M. Tohyama, Z. Phys. A335 (1990) 153.

[3] M. Tohyama and M. Gong, Z. Phys. A332 (1989) 269.

[4] M. Tohyama and P. Schuck, Eur. Phys. J. A19 (2004) 215.

[5] M. Tohyama, S. Takahara and P. Schuck, nucl-th/0311016, Eur. Phys. J. A in press.

[6] J. Sawicki, Phys. Rev. 126 (1962) 2231; J. Da Providencia, Nucl. Phys. 61 (1965) 87.

[7] H. J. Lipkin, N. Meshkov and A. J. Glick, Nucl. Phys. 62 (1965) 188.

[8] M. Tohyama and P. Schuck, Eur. Phys. J. A19 (2004) 203.

[9] M. Tohyama, Prog. Theor. Phys. 92 (1994) 905.

[10] K. Takayanagi, K. Shimizu and A. Arima, Nucl. Phys. A477 (1988) 205. 